\title{
Folding of the Chaotic General Tree and it's Chains
}

\author{
Mabrouk El-Ghoul (Corresponding author) \\ Mathematics department, Faculty of Science, Tanta University, Tanta, Egypt \\ Tel: 20-10-468-4812 E-mail: m_elghoul_2009@ @otmail.com \\ Shereen Adel Mousa \\ Mathematics department, Faculty of Girl, Ain shams University, Egypt \\ Tel: 20-16-6014-9898Ｅ-mail: shereen.3010@hotmail.com
}

Received: May 23, 2011 Accepted: June 9, 2011 Published: November 1, 2011

doi:10.5539/jmr.v3n4p118 URL: http://dx.doi.org/10.5539/jmr.v3n4p118

\begin{abstract}
In this paper, we will introduce the folding of the chaotic general tree and it's chains. The variation of the adjacent, incidence, area, volume, edge area, edge volume, area volume matrices of the chaotic general tree under the folding are discussed.The relation between the folding of the chaotic general tree and it's chains will be deduced.
\end{abstract}

AMS Subject Classification 2000 : 51H10, 57N10.

Keywords: Folding, Chaotic chains, Chaotic general tree

\section{Definitions and Background}

\subsection{Folding}

Let $f: G \rightarrow G^{\prime}$ be a map between any two graphs $G$ and $G^{\prime}$ (not necessary to be simple ) such that if $(u, v) \in$ $G,(f(u), f(v)) \in G^{\prime}$.Then $\mathrm{f}$ is called a "topological folding " of $G$ into $G^{\prime}$ provided that $d(f(u), f(v)) \leq d(u, v)$. [M. El-Ghoul and T. Homoda, 2006].

\subsection{Chaotic chains}

Let $G$ be a graph with vertices $V=\left\{v^{1}, v^{2}, \ldots v^{i}\right\}$, edges $E=\left\{e^{1}, e^{2}, \ldots e^{j}\right\}$, areas $A=\left\{a^{1}, a^{2}, \ldots a^{m}\right\}$, and volumes $L=$ $\left\{l^{1}, l^{2}, \ldots l^{n}\right\}$ carries infinite number of

chaotics [M. El-Ghoul and Sh.A.Mousa, under press].

*The $\underline{0 \text {-chaotic chains }}$ on $G$ is a system of a formal sum

$$
\left\{\begin{array}{c}
\lambda_{1}^{1 h} v_{1 h}^{1}+\lambda_{2}^{1 h} v_{1 h}^{2}+\ldots+\lambda_{i}^{1 h} v_{1 h}^{i} \\
\lambda_{1}^{2 h} v_{2 h}^{1}+\lambda_{2}^{2 h} v_{2 h}^{2}+\ldots+\lambda_{i}^{2 h} v_{2 h}^{i} \\
\cdot \\
\lambda_{1}^{\infty h} v_{\infty h}^{1}+\lambda_{2}^{\infty h} v_{\infty h}^{2}+\ldots+\lambda_{i}^{\infty h} v_{\infty h}^{i}
\end{array}\right\}
$$

where each $\lambda_{i}^{r h}$ is an integer.

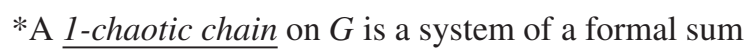

$$
\left\{\begin{array}{c}
\lambda_{1}^{1 h} e_{1 h}^{1}+\lambda_{2}^{1 h} e_{1 h}^{2}+\ldots+\lambda_{j}^{1 h} e_{1 h}^{j} \\
\lambda_{1}^{2 h} e_{2 h}^{1}+\lambda_{2}^{2 h} e_{2 h}^{2}+\ldots+\lambda_{j}^{2 h} e_{2 h}^{j} \\
\cdot \\
\lambda_{1}^{\infty h} e_{\infty h}^{1}+\lambda_{2}^{\infty h} e_{\infty h}^{2}+\ldots+\lambda_{j}^{\infty h} e_{\infty h}^{i}
\end{array}\right\}
$$


where each $\lambda_{j}^{r h}$ is an integer.

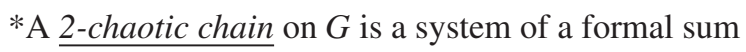

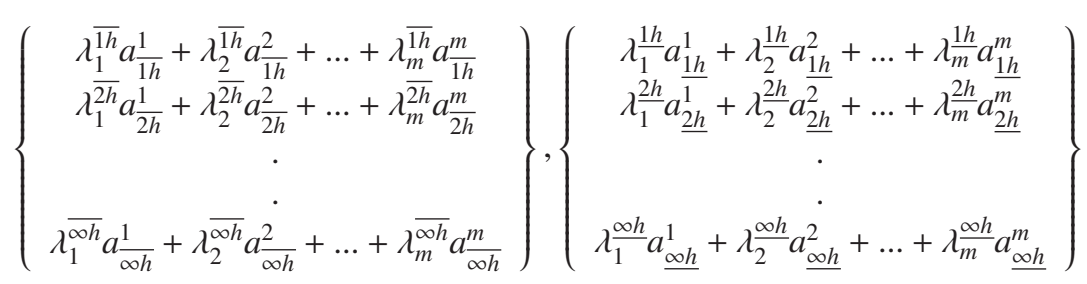

such that each $\lambda_{m}^{r h}$ is an integer and the upper bar denote that the chaotics are up and the lower bar denote that the chaotics are down the area.

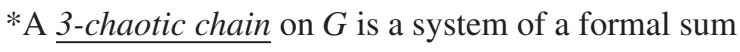

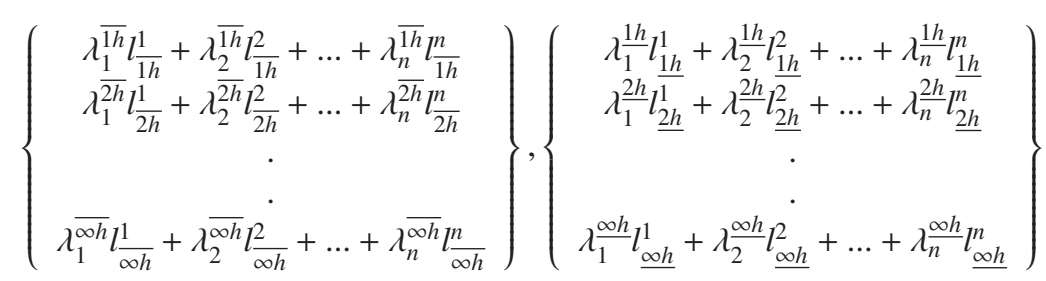

such that each $\lambda_{n}^{r h}$ is an integer and the upper bar denote that the chaotics are outside the volume and the lower bar denote that the chaotics are inside the volume.

\subsection{Chaotic General Tree}

A "Chaotic general tree" is a genaral tree that carries many physical characters.[M. El-Ghoul and Sh.A.Mousa, under press].

See Fig.(1).

Where the chaotic adjacent matrix is

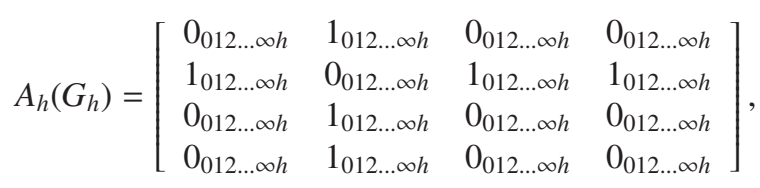

the chaotic edge matrix

$$
E_{h}\left(G_{h}\right)=\left[\begin{array}{ccc}
0_{012 \ldots \infty h} & 1_{012 \ldots \infty h} & 1_{012 \ldots \infty h} \\
1_{012 \ldots \infty h} & 0_{012 \ldots \infty h} & 1_{012 \ldots \infty h} \\
1_{012 \ldots \infty h} & 1_{012 \ldots \infty h} & 0_{012 \ldots \infty h}
\end{array}\right],
$$

the chaotic area matrix

$$
R_{h}\left(G_{h}\right)=\left[0_{012 \ldots \infty h}\right],
$$

the chaotic volume matrix

$$
V_{h}\left(G_{h}\right)=\left[0_{012 \ldots \infty h}\right],
$$

the chaotic incidence matrix 


$$
I_{h}\left(G_{h}\right)=\left[\begin{array}{ccc}
1_{012 \ldots \infty h} & 0_{012 \ldots \infty h} & 0_{012 \ldots \infty h} \\
1_{012 \ldots \infty h} & 1_{012 \ldots \infty h} & 1_{012 \ldots \infty h} \\
0_{012 \ldots \infty h} & 1_{012 \ldots \infty h} & 0_{012 \ldots \infty h} \\
0_{012 \ldots \infty h} & 0_{012 \ldots \infty h} & 1_{012 \ldots \infty h}
\end{array}\right]
$$

the chaotic vertex area matrix

$$
M_{h}\left(G_{h}\right)=\left[\begin{array}{c}
0_{012 \ldots \infty h} \\
0_{012 \ldots \infty h} \\
0_{012 \ldots \infty h} \\
1_{012 \ldots \infty h}
\end{array}\right]
$$

the chaotic vertex volume matrix

$$
N_{h}\left(G_{h}\right)=\left[\begin{array}{c}
0_{012 \ldots \infty h} \\
0_{012 \ldots \infty h} \\
1_{012 \ldots \infty h} \\
0_{012 \ldots \infty h}
\end{array}\right]
$$

the chaotic edges area matrix

$$
H_{h}\left(G_{h}\right)=\left[\begin{array}{c}
0_{012 \ldots \infty h} \\
0_{012 \ldots \infty h} \\
1_{012 \ldots \infty h}
\end{array}\right],
$$

the chaotic edge volume matrix

$$
J_{h}\left(G_{h}\right)=\left[\begin{array}{l}
0_{012 \ldots \infty h} \\
1_{012 \ldots \infty h} \\
0_{012 \ldots \infty h}
\end{array}\right],
$$

and the chaotic area volume matrix

$$
U_{h}\left(G_{h}\right)=\left[0_{012 \ldots \infty h}\right]
$$

where the lower suffix $(012 \ldots \infty h)$ refers to the existance of the chaotics, also its

0-chaotic chains are $\left\{\begin{array}{c}\lambda_{1}^{1 h} v_{1 h}^{0}+\lambda_{2}^{1 h} v_{1 h}^{1}+\lambda_{3}^{1 h} v_{1 h}^{2}+\lambda_{4}^{1 h} v_{1 h}^{3} \\ \lambda_{1}^{2 h} v_{2 h}^{0}+\lambda_{2}^{2 h} v_{2 h}^{1}+\lambda_{3}^{2 h} v_{2 h}^{2}+\lambda_{4}^{2 h} v_{2 h}^{3} \\ \cdot \\ \cdot \\ \lambda_{1}^{\infty h} v_{\infty h}^{0}+\lambda_{2}^{\infty h} v_{\infty h}^{1}+\lambda_{3}^{\infty h} v_{\infty h}^{2}+\lambda_{4}^{\infty h} v_{\infty h}^{3}\end{array}\right\}$,

the 1- chaotic chains are $\left\{\begin{array}{c}\lambda_{1}^{1 h} e_{1 h}^{0}+\lambda_{2}^{1 h} e_{1 h}^{1}+\lambda_{3}^{1 h} e_{1 h}^{2} \\ \lambda_{1}^{2 h} e_{2 h}^{0}+\lambda_{2}^{2 h} e_{2 h}^{1}+\lambda_{3}^{2 h} e_{2 h}^{2} \\ \cdot \\ \lambda_{1}^{\infty h} e_{\infty h}^{0}+\lambda_{2}^{\infty h} e_{\infty h}^{1}+\lambda_{3}^{\infty h} e_{\infty h}^{2}\end{array}\right\}$,

the 2- chaotic chains are $\left\{\begin{array}{c}\lambda_{1}^{\overline{1 h}} a_{\overline{1 h}}^{0} \\ \lambda_{1}^{\overline{2 h}} a_{\overline{2 h}}^{0} \\ \cdot \\ \lambda_{1}^{\bar{\infty}} a_{\overline{\infty h}}^{0}\end{array}\right\},\left\{\begin{array}{c}\lambda_{1}^{\frac{1 h}{a}} a_{\frac{1 h}{0}}^{0} \\ \lambda_{1}^{\frac{2 h}{\underline{2}} a_{\underline{2 h}}^{0}} \\ \cdot \\ \lambda_{1}^{\frac{\infty h}{1}} a_{\underline{\infty h}}^{0}\end{array}\right\}$, 


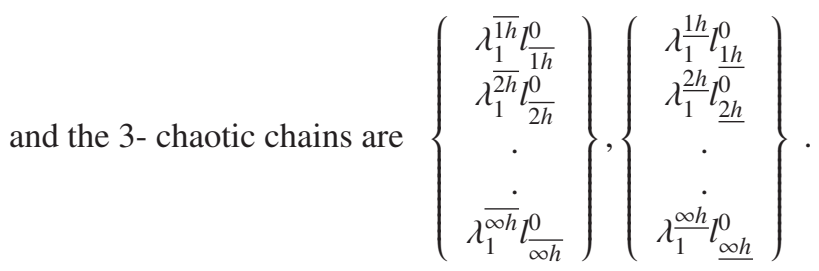

\section{The main results}

Aiming to our study, we will introduce the Folding of the chaotics of the chaotic general tree :

\subsection{Folding of the chaotics of the chaotic general tree}

Case(1):

In this case, the folding gives the original chaotic general tree without any change. All it's matrices and chaotic chains will remain as it is. It is the identity folding.

See Fig.(2).

Case(2):

In this case, the folding acts on the chaotics of the vertices $v^{0}, v^{1}, v^{2}, v^{3}$, edges $e^{0}, e^{1}, e^{2}$, area $a^{0}$, and volume $l^{0}$. The limit of the foldings is the 1-chaotic general tree (fuzzy general tree).

See Fig.(3).

Where

$$
\begin{aligned}
& * A_{h}\left(G_{h}\right)=\left[\begin{array}{cccc}
0_{012 \ldots \infty h} & 1_{012 \ldots \infty h} & 0_{012 \ldots \infty h} & 0_{012 \ldots \infty h} \\
1_{012 \ldots \infty h} & 0_{012 \ldots \infty h} & 1_{012 \ldots \infty h} & 1_{012 \ldots \infty h} \\
0_{012 \ldots \infty h} & 1_{012 \ldots \infty h} & 0_{012 \ldots \infty h} & 0_{012 \ldots \infty h} \\
0_{012 \ldots \infty h} & 1_{012 \ldots \infty h} & 0_{012 \ldots \infty h} & 0_{012 \ldots \infty h}
\end{array}\right] \stackrel{F_{21}}{\longrightarrow} \\
& A_{h}\left(F_{21}\left(G_{h}\right)\right)=\left[\begin{array}{cccc}
0_{012 \ldots(\infty-1) h} & 1_{012 \ldots(\infty-1) h} & 0_{012 \ldots(\infty-1) h} & 0_{012 \ldots(\infty-1) h} \\
1_{012 \ldots(\infty-1) h} & 0_{012 \ldots(\infty-1) h} & 1_{012 \ldots(\infty-1) h} & 1_{012 \ldots(\infty-1) h} \\
0_{012 \ldots(\infty-1) h} & 1_{012 \ldots(\infty-1) h} & 0_{012 \ldots(\infty-1) h} & 0_{012 \ldots(\infty-1) h} \\
0_{012 \ldots(\infty-1) h} & 1_{012 \ldots(\infty-1) h} & 0_{012 \ldots(\infty-1) h} & 0_{012 \ldots(\infty-1) h}
\end{array}\right]
\end{aligned}
$$

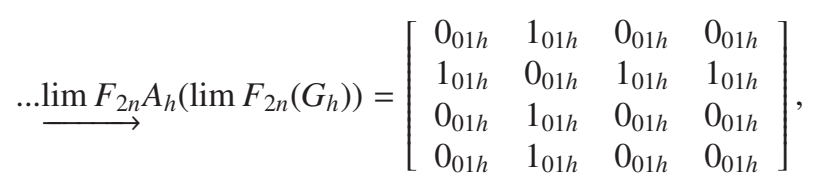

$$
\begin{aligned}
& * E_{h}\left(G_{h}\right)=\left[\begin{array}{ccc}
0_{012 \ldots \infty h} & 1_{012 \ldots \infty h} & 1_{012 \ldots \infty h} \\
1_{012 \ldots \infty h} & 0_{012 \ldots \infty h} & 1_{012 \ldots \infty h} \\
1_{012 \ldots \infty h} & 1_{012 \ldots \infty h} & 0_{012 \ldots \infty h}
\end{array}\right] \stackrel{F_{21}}{\longrightarrow} \\
& E_{h}\left(F_{21}\left(G_{h}\right)\right)=\left[\begin{array}{ccc}
0_{012 \ldots(\infty-1) h} & 1_{012 \ldots(\infty-1) h} & 1_{012 \ldots(\infty-1) h} \\
1_{012 \ldots(\infty-1) h} & 0_{012 \ldots(\infty-1) h} & 1_{012 \ldots(\infty-1) h} \\
1_{012 \ldots(\infty-1) h} & 1_{012 \ldots(\infty-1) h} & 0_{012 \ldots(\infty-1) h}
\end{array}\right] \\
& \stackrel{\lim F_{2 n}}{\longrightarrow} E_{h}\left(\lim . F_{2 n}\left(G_{h}\right)\right)=\left[\begin{array}{lll}
0_{01 h} & 1_{01 h} & 1_{01 h} \\
1_{01 h} & 0_{01 h} & 1_{01 h} \\
1_{01 h} & 1_{01 h} & 0_{01 h}
\end{array}\right], \\
& * R_{h}\left(G_{h}\right)=\left[0_{012 \ldots \infty h}\right] \stackrel{F_{21}}{\longrightarrow} R_{h}\left(F_{21}\left(G_{h}\right)\right)=\left[0_{012 \ldots(\infty-1) h}\right]
\end{aligned}
$$

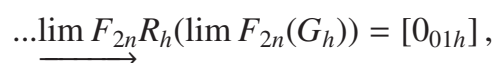

$$
\begin{aligned}
& * V_{h}\left(G_{h}\right)=\left[0_{012 \ldots \infty h}\right] \stackrel{F_{21}}{\longrightarrow} V_{h}\left(F_{21}\left(G_{h}\right)\right)=\left[0_{012 \ldots(\infty-1) h}\right]
\end{aligned}
$$




$$
\begin{aligned}
& \underset{\lim F_{2 n}}{\longrightarrow} V_{h}\left(\lim F_{2 n}\left(G_{h}\right)\right)=\left[0_{01 h}\right], \\
& * I_{h}\left(G_{h}\right)=\left[\begin{array}{ccc}
1_{012 \ldots \infty h} & 0_{012 \ldots \infty h} & 0_{012 \ldots \infty h} \\
1_{012 \ldots \infty h} & 1_{012 \ldots \infty h} & 1_{012 \ldots \infty h} \\
0_{012 \ldots \infty h} & 1_{012 \ldots \infty h} & 0_{012 \ldots \infty h} \\
0_{012 \ldots \infty h} & 0_{012 \ldots \infty h} & 1_{012 \ldots \infty h}
\end{array}\right] \stackrel{F_{21}}{\longrightarrow} \\
& I_{h}\left(F_{21}\left(G_{h}\right)\right)=\left[\begin{array}{ccc}
1_{012 \ldots(\infty-1) h} & 0_{012 \ldots(\infty-1) h} & 0_{012 \ldots(\infty-1) h} \\
1_{012 \ldots(\infty-1) h} & 1_{012 \ldots(\infty-1) h} & 1_{012 \ldots(\infty-1) h} \\
0_{012 \ldots(\infty-1) h} & 1_{012 \ldots(\infty-1) h} & 0_{012 \ldots(\infty-1) h} \\
0_{012 \ldots(\infty-1) h} & 0_{012 \ldots(\infty-1) h} & 1_{012 \ldots(\infty-1) h}
\end{array}\right] \\
& \underset{\lim F_{2 n} I_{h}}{\longrightarrow}\left(\lim F_{2 n}\left(G_{h}\right)\right)=\left[\begin{array}{ccc}
1_{01 h} & 0_{01 h} & 0_{01 h} \\
1_{01 h} & 1_{01 h} & 1_{01 h} \\
0_{01 h} & 1_{01 h} & 0_{01 h} \\
0_{01 h} & 0_{01 h} & 1_{01 h}
\end{array}\right], \\
& * M_{h}\left(G_{h}\right)=\left[\begin{array}{c}
0_{012 \ldots \infty h} \\
0_{012 \ldots \infty} \\
0_{012 \ldots \infty h} \\
1_{012 \ldots \infty h}
\end{array}\right] \stackrel{F_{21}}{\longrightarrow} M_{h}\left(F_{21}\left(G_{h}\right)\right)=\left[\begin{array}{c}
0_{012 \ldots(\infty-1) h} \\
0_{012 \ldots(\infty-1) h} \\
0_{012 \ldots(\infty-1) h} \\
1_{012 \ldots(\infty-1) h}
\end{array}\right] \\
& \stackrel{\lim F_{2 n}}{\longrightarrow} M_{h}\left(\lim F_{2 n}\left(G_{h}\right)\right)=\left[\begin{array}{c}
0_{01 h} \\
0_{01 h} \\
0_{01 h} \\
1_{01 h}
\end{array}\right] \\
& * N_{h}\left(G_{h}\right)=\left[\begin{array}{c}
0_{012 \ldots \infty h} \\
0_{012 \ldots \infty h} \\
1_{012 \ldots \infty h} \\
0_{012 \ldots \infty h}
\end{array}\right] \underset{F_{21}}{\longrightarrow} N_{h}\left(F_{21}\left(G_{h}\right)\right)=\left[\begin{array}{c}
0_{012 \ldots(\infty-1) h} \\
0_{012 \ldots(\infty-1) h} \\
1_{012 \ldots(\infty-1) h} \\
0_{012 \ldots(\infty-1) h}
\end{array}\right] \\
& \underset{\lim F_{2 n}}{\longrightarrow} N_{h}\left(\lim F_{2 n}\left(G_{h}\right)\right)=\left[\begin{array}{c}
0_{01 h} \\
0_{01 h} \\
1_{01 h} \\
0_{01 h}
\end{array}\right] \\
& * H_{h}\left(G_{h}\right)=\left[\begin{array}{c}
0_{012 \ldots \infty h} \\
0_{012 \ldots \infty h} \\
1_{012 \ldots \infty h}
\end{array}\right] \stackrel{F_{21}}{\longrightarrow} H_{h}\left(F_{21}\left(G_{h}\right)\right)=\left[\begin{array}{c}
0_{012 \ldots(\infty-1) h} \\
0_{012 \ldots(\infty-1) h} \\
1_{012 \ldots(\infty-1) h}
\end{array}\right] \\
& \underset{\lim F_{2 n}}{\longrightarrow} H_{h}\left(\lim F_{2 n}\left(G_{h}\right)\right)=\left[\begin{array}{c}
0_{01 h} \\
0_{01 h} \\
1_{01 h}
\end{array}\right], \\
& * J_{h}\left(G_{h}\right)=\left[\begin{array}{c}
0_{012 \ldots \infty h} \\
1_{012 \ldots \infty h} \\
0_{012 \ldots \infty h}
\end{array}\right] \underset{F_{21}}{\longrightarrow} J_{h}\left(F_{21}\left(G_{h}\right)\right)=\left[\begin{array}{c}
0_{012 \ldots(\infty-1) h} \\
1_{012 \ldots(\infty-1) h} \\
0_{012 \ldots(\infty-1) h}
\end{array}\right] \\
& \underset{\lim F_{2 n}}{\longrightarrow} J_{h}\left(\lim F_{2 n}\left(G_{h}\right)\right)=\left[\begin{array}{c}
0_{01 h} \\
1_{01 h} \\
0_{01 h}
\end{array}\right] \\
& * U_{h}\left(G_{h}\right)=\left[0_{012 \ldots \infty h}\right] \underset{F_{21}}{\longrightarrow} U_{h}\left(F_{21}\left(G_{h}\right)\right)=\left[0_{012 \ldots(\infty-1) h}\right]
\end{aligned}
$$




$$
\stackrel{\lim F_{2 n}}{\longrightarrow} U_{h}\left(\lim F_{2 n}\left(G_{h}\right)\right)=\left[0_{01 h}\right],
$$

and the 0-chaotic chain are

$$
\begin{aligned}
& \left\{\begin{array}{c}
\lambda_{1}^{1 h} v_{1 h}^{0}+\lambda_{2}^{1 h} v_{1 h}^{1}+\lambda_{3}^{1 h} v_{1 h}^{2}+\lambda_{4}^{1 h} v_{1 h}^{3} \\
\lambda_{1}^{2 h} v_{2 h}^{0}+\lambda_{2}^{2 h} v_{2 h}^{1}+\lambda_{3}^{2 h} v_{2 h}^{2}+\lambda_{4}^{2 h} v_{2 h}^{3} \\
\cdot \\
\cdot \\
\lambda_{1}^{\infty h} v_{\infty h}^{0}+\lambda_{2}^{\infty h} v_{\infty h}^{1}+\lambda_{3}^{\infty h} v_{\infty h}^{2}+\lambda_{4}^{\infty h} v_{\infty h}^{3}
\end{array}\right\} \stackrel{F_{21}}{\longrightarrow}\left\{\begin{array}{c}
\lambda_{1}^{1 h} v_{1 h}^{0}+\lambda_{2}^{1 h} v_{1 h}^{1}+\lambda_{3}^{1 h} v_{1 h}^{2}+\lambda_{4}^{1 h} v_{1 h}^{3} \\
\lambda_{1}^{2 h} v_{2 h}^{0}+\lambda_{2}^{2 h} v_{2 h}^{1}+\lambda_{3}^{2 h} v_{2 h}^{2}+\lambda_{4}^{2 h} v_{2 h}^{3} \\
\\
\\
\\
\lambda_{1}^{(\infty-1) h} v_{(\infty-1) h}^{0}+\lambda_{2}^{(\infty-1) h} v_{(\infty-1) h}^{1} \\
+\lambda_{3}^{(\infty-1) h} v_{(\infty-1) h}^{2}+\lambda_{4}^{(\infty-1) h} v_{(\infty-1) h}^{3}
\end{array}\right\} \\
& \stackrel{\lim F_{2 n}}{\longrightarrow} \lambda_{1}^{1 h} v_{1 h}^{0}+\lambda_{2}^{1 h} v_{1 h}^{1}+\lambda_{3}^{1 h} v_{1 h}^{2}+\lambda_{4}^{1 h} v_{1 h}^{3},
\end{aligned}
$$

the 1 - chaotic chains are

$$
\begin{aligned}
& \left\{\begin{array}{c}
\lambda_{1}^{1 h} e_{1 h}^{0}+\lambda_{2}^{1 h} e_{1 h}^{1}+\lambda_{3}^{1 h} e_{1 h}^{2} \\
\lambda_{1}^{2 h} e_{2 h}^{0}+\lambda_{2}^{2 h} e_{2 h}^{1}+\lambda_{3}^{2 h} e_{2 h}^{2} \\
\cdot \\
\lambda_{1}^{\infty h} e_{\infty h}^{0}+\lambda_{2}^{\infty h} e_{\infty h}^{1}+\lambda_{3}^{\infty h} e_{\infty h}^{2}
\end{array}\right\} \stackrel{F_{21}}{\longrightarrow}\left\{\begin{array}{c}
\lambda_{1}^{1 h} e_{1 h}^{0}+\lambda_{2}^{1 h} e_{1 h}^{1}+\lambda_{3}^{1 h} e_{1 h}^{2} \\
\lambda_{1}^{2 h} e_{2 h}^{0}+\lambda_{2}^{2 h} e_{2 h}^{1 h}+\lambda_{3}^{2 h} e_{2 h}^{2 h} \\
\cdot \\
\cdot \\
\lambda_{1}^{(\infty-1) h} e_{(\infty-1) h}^{0}+\lambda_{2}^{(\infty-1) h} e_{(\infty-1) h}^{1}+\lambda_{3}^{(\infty-1) h} e_{(\infty-1) h}^{2}
\end{array}\right\} \\
& \stackrel{\lim F_{2 n}}{\longrightarrow} \lambda_{1}^{1 h} e_{1 h}^{0}+\lambda_{2}^{1 h} e_{1 h}^{1}+\lambda_{3}^{1 h} e_{1 h}^{2}
\end{aligned}
$$

the 2- chaotic chains are

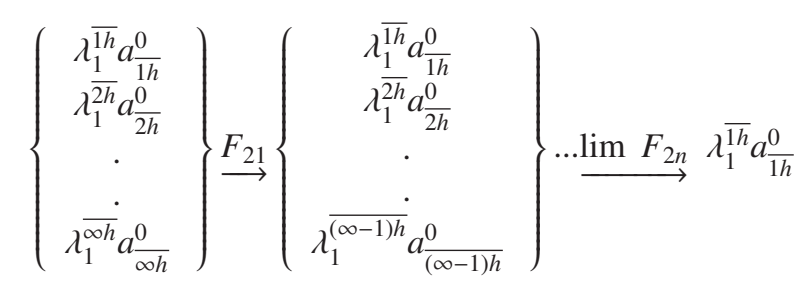

and

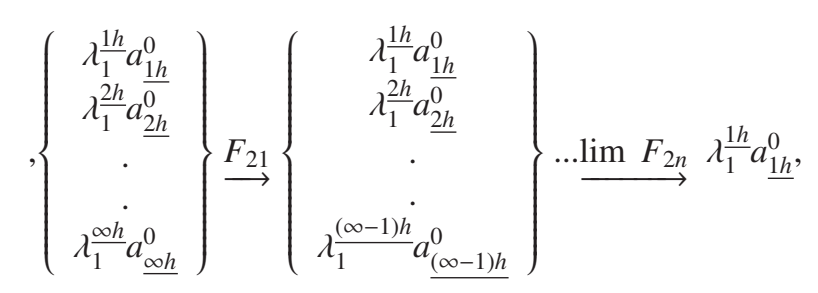

and the 3- chaotic chains are

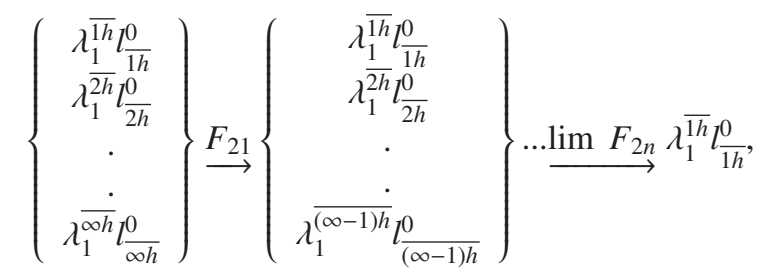

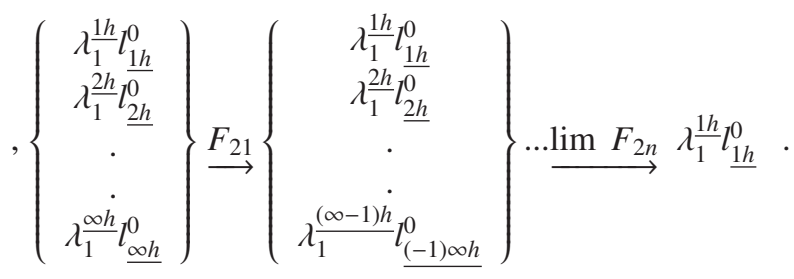




\section{Case(3):}

Here the chaotic on the vertices $v^{0}, v^{1}, v^{2}, v^{3}$ will be reduce, such that $F_{3 m}\left(v_{j h}^{i}\right)=v_{(j-1) h}^{i}$, where $i=0,1,2,3, j=0,1,2 \ldots \infty$ , $m=1, \ldots n$.The adjacent $A_{h}\left(G_{h}\right)$, incident $I_{h}\left(G_{h}\right)$, vertex area $M_{h}\left(G_{h}\right)$, and vertex volume $N_{h}\left(G_{h}\right)$ matrices will change, but the edge $E_{h}\left(G_{h}\right)$, area $R_{h}\left(G_{h}\right)$, volume $V_{h}\left(G_{h}\right)$, edge area $H_{h}\left(G_{h}\right)$,edge volume $J_{h}\left(G_{h}\right)$ matrices, and area volume $U_{h}\left(G_{h}\right)$ matreices will not change.

See Fig.(4).

$$
A_{h}\left(F_{31}\left(G_{h}\right)\right)=\left[\begin{array}{cccc}
0_{012 \ldots(\infty-1) h} & 1_{012 \ldots(\infty-1) h} & 0_{012 \ldots(\infty-1) h} & 0_{012 \ldots(\infty-1) h} \\
1_{012 \ldots(\infty-1) h} & 0_{012 \ldots(\infty-1) h} & 1_{012 \ldots(\infty-1) h} & 1_{012 \ldots(\infty-1) h} \\
0_{012 \ldots(\infty-1) h} & 1_{012 \ldots(\infty-1) h} & 0_{012 \ldots(\infty-1) h} & 0_{012 \ldots(\infty-1) h} \\
0_{012 \ldots(\infty-1) h} & 1_{012 \ldots(\infty-1) h} & 0_{012 \ldots(\infty-1) h} & 0_{012 \ldots(\infty-1) h}
\end{array}\right]
$$

$$
\begin{aligned}
& \stackrel{\lim F_{3 n}}{\longrightarrow} A_{h}\left(\lim F_{3 n}\left(G_{h}\right)\right)=\left[\begin{array}{cccc}
0_{01 h} & 1_{01 h} & 0_{01 h} & 0_{01 h} \\
1_{01 h} & 0_{01 h} & 1_{01 h} & 1_{01 h} \\
0_{01 h} & 1_{01 h} & 0_{01 h} & 0_{01 h} \\
0_{01 h} & 1_{01 h} & 0_{01 h} & 0_{01 h}
\end{array}\right], \\
& * I_{h}\left(G_{h}\right)=\left[\begin{array}{ccc}
1_{012 \ldots \infty h} & 0_{012 \ldots \infty h} & 0_{012 \ldots \infty h} \\
1_{012 \ldots \infty h} & 1_{012 \ldots \infty h} & 1_{012 \ldots \infty h} \\
0_{012 \ldots \infty h} & 1_{012 \ldots \infty h} & 0_{012 \ldots \infty h} \\
0_{012 \ldots \infty h} & 0_{012 \ldots \infty h} & 1_{012 \ldots \infty h}
\end{array}\right] \stackrel{F_{31}}{\longrightarrow} \\
& I_{h}\left(F_{31}\left(G_{h}\right)\right)=\left[\begin{array}{lll}
1_{012 \ldots(\infty-1) h} & 0_{012 \ldots(\infty-1) h} & 0_{012 \ldots(\infty-1) h} \\
1_{012 \ldots(\infty-1) h} & 1_{012 \ldots(\infty-1) h} & 1_{012 \ldots(\infty-1) h} \\
0_{012 \ldots(\infty-1) h} & 1_{012 \ldots(\infty-1) h} & 0_{012 \ldots(\infty-1) h} \\
0_{012 \ldots(\infty-1) h} & 0_{012 \ldots(\infty-1) h} & 1_{012 \ldots(\infty-1) h}
\end{array}\right] \\
& \underset{\lim F_{3 n}}{\longrightarrow} I_{h}\left(\lim F_{3 n}\left(G_{h}\right)\right)=\left[\begin{array}{ccc}
1_{01 h} & 0_{01 h} & 0_{01 h} \\
1_{01 h} & 1_{01 h} & 1_{01 h} \\
0_{01 h} & 1_{01 h} & 0_{01 h} \\
0_{01 h} & 0_{01 h} & 1_{01 h}
\end{array}\right], \\
& * M_{h}\left(G_{h}\right)=\left[\begin{array}{c}
0_{012 \ldots \infty h} \\
0_{012 \ldots \infty} \\
0_{012 \ldots \infty h} \\
1_{012 \ldots \infty h}
\end{array}\right] \stackrel{F_{31}}{\longrightarrow} M_{h}\left(F_{31}\left(G_{h}\right)=\left[\begin{array}{c}
0_{012 \ldots(\infty-1) h} \\
0_{012 \ldots(\infty-1) h} \\
0_{012 \ldots(\infty-1) h} \\
1_{012 \ldots(\infty-1) h}
\end{array}\right]\right. \\
& \stackrel{\lim F_{3 n}}{\longrightarrow} M_{h}\left(\lim F_{3 n}\left(G_{h}\right)\right)=\left[\begin{array}{c}
0_{01 h} \\
0_{01 h} \\
0_{01 h} \\
1_{01 h}
\end{array}\right], \\
& * N_{h}\left(G_{h}\right)=\left[\begin{array}{c}
0_{012 \ldots \infty h} \\
0_{012 \ldots \infty h} \\
1_{012 \ldots \infty h} \\
0_{012 \ldots \infty h}
\end{array}\right] \underset{F_{31}}{\longrightarrow} N_{h}\left(F_{31}\left(G_{h}\right)\right)=\left[\begin{array}{c}
0_{012 \ldots(\infty-1) h} \\
0_{012 \ldots(\infty-1) h} \\
1_{012 \ldots(\infty-1) h} \\
0_{012 \ldots(\infty-1) h}
\end{array}\right] \\
& \underset{\lim F_{3 n}}{\longrightarrow} N_{h}\left(\lim F_{3 n}\left(G_{h}\right)\right)=\left[\begin{array}{c}
0_{01 h} \\
0_{01 h} \\
1_{01 h} \\
0_{01 h}
\end{array}\right]
\end{aligned}
$$

The 1- chaotic, 2- chasotic, 3 - chaotic chains will not change. Only the 0 - chaotic chain will change as follows

$$
\left\{\begin{array}{c}
\lambda_{1}^{1 h} v_{1 h}^{0}+\lambda_{2}^{1 h} v_{1 h}^{1}+\lambda_{3}^{1 h} v_{1 h}^{2}+\lambda_{4}^{1 h} v_{1 h}^{3} \\
\lambda_{1}^{2 h} v_{2 h}^{0}+\lambda_{2}^{2 h} v_{2 h}^{1}+\lambda_{3}^{2 h} v_{2 h}^{2}+\lambda_{4}^{2 h} v_{2 h}^{3} \\
\cdot \\
\lambda_{1}^{\infty h} v_{\infty h}^{0}+\lambda_{2}^{\infty h} v_{\infty h}^{1}+\lambda_{3}^{\infty h} v_{\infty h}^{2}+\lambda_{4}^{\infty h} v_{\infty h}^{3}
\end{array}\right\} \stackrel{F_{31}}{\longrightarrow}\left\{\begin{array}{c}
\lambda_{1}^{1 h} v_{1 h}^{0}+\lambda_{2}^{1 h} v_{1 h}^{1}+\lambda_{3}^{1 h} v_{1 h}^{2}+\lambda_{4}^{1 h} v_{1 h}^{3} \\
\lambda_{1}^{2 h} v_{2 h}^{0}+\lambda_{2}^{2 h} v_{2 h}^{1}+\lambda_{3}^{2 h} v_{2 h}^{2}+\lambda_{4}^{2 h} v_{2 h}^{3} \\
\cdot \\
\cdot \\
\lambda_{1}^{(\infty-1) h} v_{(\infty-1) h}^{0}+\lambda_{2}^{(\infty-1) h} v_{(\infty-1) h}^{1} \\
+\lambda_{3}^{(\infty-1) h} v_{(\infty-1) h}^{2}+\lambda_{4}^{(\infty-1) h} v_{(\infty-1) h}^{3}
\end{array}\right\}
$$




$$
\underset{\ldots \lim . F_{3 n}}{\longrightarrow} \lambda_{1} v^{0}+\lambda_{2} v^{1}+\lambda_{3} v^{2}+\lambda_{4} v^{3},
$$

Case(4) :

In this case, the folding acts on the chaotics of the edgese $e^{0}, e^{1}, e^{2}$, such that $F_{4 m}\left(e_{j h}^{i}\right)=e_{(j-1) h}^{i}$, where $i=0,1,2, j=$ $0,1,2 \ldots \infty, m=1, \ldots, n$. Then the chaotic adjacent $A_{h}\left(G_{h}\right)$, edge $E_{h}\left(G_{h}\right)$, incident $I_{h}\left(G_{h}\right)$, edge area $H_{h}\left(G_{h}\right)$, edge volume $U_{h}\left(G_{h}\right)$ matrices and the 1-chaotic chains only will change.

See Fig.(5).

The matrices will be in the form

$$
\begin{aligned}
& * A_{h}\left(G_{h}\right)=\left[\begin{array}{llll}
0_{012 \ldots \infty h} & 1_{012 \ldots \infty h} & 0_{012 \ldots \infty h} & 0_{012 \ldots \infty h} \\
1_{012 \ldots \infty h} & 0_{012 \ldots \infty h} & 1_{012 \ldots \infty h} & 1_{012 \ldots \infty h} \\
0_{012 \ldots \infty h} & 1_{012 \ldots \infty h} & 0_{012 \ldots \infty h} & 0_{012 \ldots \infty h} \\
0_{012 \ldots \infty h} & 1_{012 \ldots \infty h} & 0_{012 \ldots \infty h} & 0_{012 \ldots \infty h}
\end{array}\right] \stackrel{F_{41}}{\longrightarrow} \\
& A_{h}\left(F_{41}\left(G_{h}\right)\right)=\left[\begin{array}{cccc}
0_{012 \ldots(\infty-1) h} & 1_{012 \ldots(\infty-1) h} & 0_{012 \ldots(\infty-1) h} & 0_{012 \ldots(\infty-1) h} \\
1_{012 \ldots(\infty-1) h} & 0_{012 \ldots(\infty-1) h} & 1_{012 \ldots(\infty-1) h} & 1_{012 \ldots(\infty-1) h} \\
0_{012 \ldots(\infty-1) h} & 1_{012 \ldots(\infty-1) h} & 0_{012 \ldots(\infty-1) h} & 0_{012 \ldots(\infty-1) h} \\
0_{012 \ldots(\infty-1) h} & 1_{012 \ldots(\infty-1) h} & 0_{012 \ldots(\infty-1) h} & 0_{012 \ldots(\infty-1) h}
\end{array}\right] \\
& \stackrel{\lim F_{4 n}}{\longrightarrow} A_{h}\left(\lim F_{4 n}\left(G_{h}\right)\right)=\left[\begin{array}{cccc}
0_{01 h} & 1_{01 h} & 0_{01 h} & 0_{01 h} \\
1_{01 h} & 0_{01 h} & 1_{01 h} & 1_{01 h} \\
0_{01 h} & 1_{01 h} & 0_{01 h} & 0_{01 h} \\
0_{01 h} & 1_{01 h} & 0_{01 h} & 0_{01 h}
\end{array}\right], \\
& * E_{h}\left(G_{h}\right)=\left[\begin{array}{ccc}
0_{012 \ldots \infty h} & 1_{012 \ldots \infty h} & 1_{012 \ldots \infty h} \\
1_{012 \ldots \infty h} & 0_{012 \ldots \infty h} & 1_{012 \ldots \infty h} \\
1_{012 \ldots \infty h} & 1_{012 \ldots \infty h} & 0_{012 \ldots \infty h}
\end{array}\right] \stackrel{F_{41}}{\longrightarrow} \\
& E_{h}\left(F_{41}\left(G_{h}\right)\right)=\left[\begin{array}{ccc}
0_{012 \ldots(\infty-1) h} & 1_{012 \ldots(\infty-1) h} & 1_{012 \ldots(\infty-1) h} \\
1_{012 \ldots(\infty-1) h} & 0_{012 \ldots(\infty-1) h} & 1_{012 \ldots(\infty-1) h} \\
1_{012 \ldots(\infty-1) h} & 1_{012 \ldots(\infty-1) h} & 0_{012 \ldots(\infty-1) h}
\end{array}\right] \\
& \underset{\lim F_{4 n}}{\longrightarrow} E_{h}\left(\lim F_{4 n}\left(G_{h}\right)\right)=\left[\begin{array}{ccc}
0_{01 h} & 1_{01 h} & 1_{01 h} \\
1_{01 h} & 0_{01 h} & 1_{01 h} \\
1_{01 h} & 1_{01 h} & 0_{01 h}
\end{array}\right], \\
& * I_{h}\left(G_{h}\right)=\left[\begin{array}{lll}
1_{012 \ldots \infty h} & 0_{012 \ldots \infty h} & 0_{012 \ldots \infty h} \\
1_{012 \ldots \infty h} & 1_{012 \ldots \infty h} & 1_{012 \ldots \infty h} \\
0_{012 \ldots \infty h} & 1_{012 \ldots \infty h} & 0_{012 \ldots \infty h} \\
0_{012 \ldots \infty h} & 0_{012 \ldots \infty h} & 1_{012 \ldots \infty h}
\end{array}\right] \stackrel{F_{41}}{\longrightarrow} \\
& I_{h}\left(F_{41}\left(G_{h}\right)\right)=\left[\begin{array}{ccc}
1_{012 \ldots(\infty-1) h} & 0_{012 \ldots(\infty-1) h} & 0_{012 \ldots(\infty-1) h} \\
1_{012 \ldots(\infty-1) h} & 1_{012 \ldots(\infty-1) h} & 1_{012 \ldots(\infty-1) h} \\
0_{012 \ldots(\infty-1) h} & 1_{012 \ldots(\infty-1) h} & 0_{012 \ldots(\infty-1) h} \\
0_{012 \ldots(\infty-1) h} & 0_{012 \ldots(\infty-1) h} & 1_{012 \ldots(\infty-1) h}
\end{array}\right] \\
& \stackrel{\lim F_{4 n}}{\longrightarrow} I_{h}\left(\lim F_{4 n}\left(G_{h}\right)\right)=\left[\begin{array}{ccc}
1_{01 h} & 0_{01 h} & 0_{01 h} \\
1_{01 h} & 1_{01 h} & 1_{01 h} \\
0_{01 h} & 1_{01 h} & 0_{01 h} \\
0_{01 h} & 0_{01 h} & 1_{01 h}
\end{array}\right], \\
& * H_{h}\left(G_{h}\right)=\left[\begin{array}{c}
0_{012 \ldots \infty h} \\
0_{012 \ldots \infty h} \\
1_{012 \ldots \infty h}
\end{array}\right] \stackrel{F_{41}}{\longrightarrow} H_{h}\left(F_{41}\left(G_{h}\right)\right)=\left[\begin{array}{c}
0_{012 \ldots(\infty-1) h} \\
0_{012 \ldots(\infty-1) h} \\
1_{012 \ldots(\infty-1) h}
\end{array}\right] \\
& \stackrel{\lim F_{4 n}}{\longrightarrow} H_{h}\left(\lim F_{4 n}\left(G_{h}\right)\right)=\left[\begin{array}{c}
0_{01 h} \\
0_{01 h} \\
1_{01 h}
\end{array}\right], \\
& * J_{h}\left(G_{h}\right)=\left[\begin{array}{c}
0_{012 \ldots \infty h} \\
1_{012 \ldots \infty h} \\
0_{012 \ldots \infty h}
\end{array}\right] \underset{F_{41}}{\longrightarrow} J_{h}\left(F_{41}\left(G_{h}\right)\right)=\left[\begin{array}{c}
0_{012 \ldots(\infty-1) h} \\
1_{012 \ldots(\infty-1) h} \\
0_{012 \ldots(\infty-1) h}
\end{array}\right]
\end{aligned}
$$




$$
\underset{\ldots \lim F_{4 n}}{\longrightarrow} J_{h}\left(\lim F_{4 n}\left(G_{h}\right)\right)=\left[\begin{array}{c}
0_{01 h} \\
1_{01 h} \\
0_{01 h}
\end{array}\right],
$$

and it's 1-chaotic chains are

$$
\begin{gathered}
\left\{\begin{array}{c}
\lambda_{1}^{1 h} e_{1 h}^{0}+\lambda_{2}^{1 h} e_{1 h}^{1}+\lambda_{3}^{1 h} e_{1 h}^{2} \\
\lambda_{1}^{2 h} e_{2 h}^{0}+\lambda_{2}^{2 h} e_{2 h}^{1}+\lambda_{3}^{2 h} e_{2 h}^{2} \\
\cdot \\
\lambda_{1}^{\infty h} e_{\infty h}^{0}+\lambda_{2}^{\infty h} e_{\infty h}^{1}+\lambda_{3}^{\infty h} e_{\infty h}^{2}
\end{array}\right\} \stackrel{F_{41}}{\longrightarrow}\left\{\begin{array}{c}
\lambda_{1}^{1 h} e_{1 h}^{0}+\lambda_{2}^{1 h} e_{1 h}^{1}+\lambda_{3}^{1 h} e_{1 h}^{2} \\
\lambda_{1}^{2 h} e_{2 h}^{0}+\lambda_{2}^{2 h} e_{2 h}^{1}+\lambda_{3}^{2 h} e_{2 h}^{2} \\
\cdot \\
\cdot \\
\lambda_{1}^{(\infty-1) h} e_{(\infty-1) h}^{0}+\lambda_{2}^{(\infty-1) h} e_{(\infty-1) h}^{1}+\lambda_{3}^{(\infty-1) h} e_{(\infty-1) h}^{2}
\end{array}\right\} \\
\stackrel{\lim F_{4 n}\left\{\lambda_{1} e^{0}+\lambda_{2} e^{1}+\lambda_{3} e^{2}\right\} .}{\longrightarrow}
\end{gathered}
$$

\section{Corollary(1) :}

The folding of the chaotic edges of a chaotic general tree leads to folding of it's chaotic vertices.

\section{Case(5) :}

In this case, we fold the chaotics of the area $a^{0}$,such that $F_{5}\left(a_{j h}^{0}\right)=a_{(j-1) h}^{0}$, where $j=0,1,2 \ldots \infty$. The matrices which will be changed are the adjacent $A_{h}\left(G_{h}\right)$, area $R_{h}\left(G_{h}\right)$, vertex area $M_{h}\left(G_{h}\right)$, edge area $H_{h}\left(G_{h}\right)$ and the area volume $U_{h}\left(G_{h}\right)$ matrices.

See Fig.(6).

$$
\begin{aligned}
& * A_{h}\left(G_{h}\right)=\left[\begin{array}{llll}
0_{012 \ldots \infty h} & 1_{012 \ldots \infty h} & 0_{012 \ldots \infty h} & 0_{012 \ldots \infty h} \\
1_{012 \ldots \infty h} & 0_{012 \ldots \infty h} & 1_{012 \ldots \infty h} & 1_{012 \ldots \infty h} \\
0_{012 \ldots \infty h} & 1_{012 \ldots \infty h} & 0_{012 \ldots \infty h} & 0_{012 \ldots \infty h} \\
0_{012 \ldots \infty h} & 1_{012 \ldots \infty h} & 0_{012 \ldots \infty h} & 0_{012 \ldots \infty h}
\end{array}\right] \stackrel{F_{51}}{\longrightarrow} \\
& A_{h}\left(F_{51}\left(G_{h}\right)\right)=\left[\begin{array}{cccc}
0_{012 \ldots \infty h} & 1_{012 \ldots \infty h} & 0_{012 \ldots \infty h} & 0_{012 \ldots \infty h} \\
1_{012 \ldots \infty h} & 0_{012 \ldots \infty h} & 1_{012 \ldots \infty h} & 1_{012 \ldots \infty h} \\
0_{012 \ldots \infty h} & 1_{012 \ldots \infty h} & 0_{012 \ldots \infty-1) h} & 0_{012 \ldots \infty h} \\
0_{012 \ldots \infty h} & 1_{012 \ldots \infty h} & 0_{012 \ldots \infty h} & 0_{012 \ldots \infty h}
\end{array}\right] \\
& \underset{\lim F_{5 n}}{\longrightarrow} A_{h}\left(\lim F_{5 n}\left(G_{h}\right)\right)=\left[\begin{array}{cccc}
0_{012 \ldots \infty h} & 1_{012 \ldots \infty h} & 0_{012 \ldots \infty h} & 0_{012 \ldots \infty h} \\
1_{012 \ldots \infty h} & 0_{012 \ldots \infty h} & 1_{012 \ldots \infty h} & 1_{012 \ldots \infty h} \\
0_{012 \ldots \infty h} & 1_{012 \ldots \infty h} & 0_{01 h} & 0_{012 \ldots \infty h} \\
0_{012 \ldots \infty h} & 1_{012 \ldots \infty h} & 0_{012 \ldots \infty h} & 0_{012 \ldots \infty h}
\end{array}\right], \\
& * R_{h}\left(G_{h}\right)=\left[0_{012 \ldots \infty h}\right] \underset{51}{\longrightarrow} R_{h}\left(F_{51}\left(G_{h}\right)\right)=\left[0_{012 \ldots(\infty-1) h}\right] \\
& \underset{\lim F_{5 n}}{\longrightarrow} R_{h}\left(\lim F_{5 n}\left(G_{h}\right)\right)=\left[0_{01 h}\right], \\
& * M_{h}\left(G_{h}\right)=\left[\begin{array}{c}
0_{012 \ldots \infty h} \\
0_{012 \ldots \infty h} \\
0_{012 \ldots \infty h} \\
1_{012 \ldots \infty h}
\end{array}\right] \stackrel{F_{51}}{\longrightarrow} M_{h}\left(F_{51}\left(G_{h}\right)\right)=\left[\begin{array}{c}
0_{012 \ldots \infty h} \\
0_{012 \ldots \infty h} \\
0_{012 \ldots(\infty-1) h} \\
1_{012 \ldots \infty h}
\end{array}\right] \\
& \stackrel{\lim F_{5 n}}{\longrightarrow} M_{h}\left(\lim F_{5 n}\left(G_{h}\right)\right)=\left[\begin{array}{c}
0_{012 \ldots \infty h} \\
0_{012 \ldots \infty h} \\
0_{01 h} \\
1_{012 \ldots \infty h}
\end{array}\right], \\
& * H_{h}\left(G_{h}\right)=\left[\begin{array}{c}
0_{012 \ldots \infty h} \\
0_{012 \ldots \infty h} \\
1_{012 \ldots \infty h}
\end{array}\right] \underset{F_{51}}{\longrightarrow} H_{h}\left(F_{51}\left(G_{h}\right)\right)=\left[\begin{array}{c}
0_{012 \ldots(\infty-1) h} \\
0_{012 \ldots(\infty-1) h} \\
1_{012 \ldots(\infty-1) h}
\end{array}\right] \\
& \underset{\lim F_{5 n}}{\longrightarrow} H_{h}\left(\lim F_{5 n}\left(G_{h}\right)\right)=\left[\begin{array}{c}
0_{01 h} \\
0_{01 h} \\
1_{01 h}
\end{array}\right]
\end{aligned}
$$


and

$$
\begin{aligned}
* U_{h}\left(G_{h}\right)=[ & {\left[0_{012 \ldots \infty h}\right] \stackrel{F_{51}}{\longrightarrow} U_{h}\left(F_{51}\left(G_{h}\right)\right)=\left[0_{012 \ldots(\infty-1) h}\right] } \\
& \quad . \lim F_{5 n} U_{h}\left(\lim F_{5 n}\left(G_{h}\right)\right)=\left[0_{01 h}\right] .
\end{aligned}
$$

It's 2- chaotic chains are

$$
\begin{aligned}
& \left\{\begin{array}{c}
\lambda_{1}^{\overline{1 h}} a_{\overline{1 h}}^{0} \\
\lambda_{1}^{\overline{2 h}} a_{\overline{2 h}}^{0} \\
\cdot \\
\lambda_{1}^{\overline{\infty h}} a_{\overline{\infty h}}^{0}
\end{array}\right\} \stackrel{F_{51}}{\longrightarrow}\left\{\begin{array}{c}
\lambda_{1}^{\overline{1 h}} a_{\overline{1 h}}^{0} \\
\lambda_{1}^{\overline{2 h}} a_{\overline{2 h}}^{0} \\
\cdot \\
\lambda_{1}^{\overline{(\infty-1) h}} a_{\overline{(\infty-1) h}}^{0}
\end{array}\right\} \stackrel{\lim F_{5 n}}{\longrightarrow}\left\{\lambda_{1} a^{0}\right\},
\end{aligned}
$$

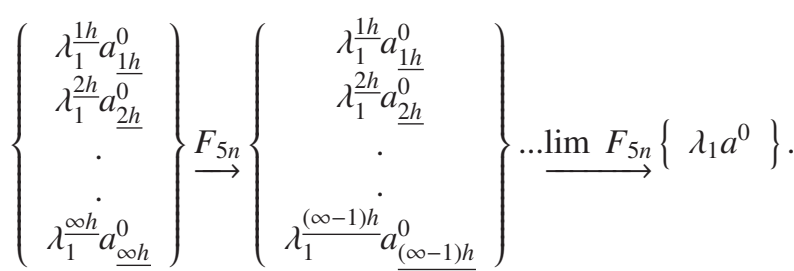

Case(6) :

Here the folding acts on the chaotic of the volume. The edge $E_{h}\left(G_{h}\right)$, area $R_{h}\left(G_{h}\right)$, vertex area $M\left(G_{h}\right)$, and edge area $H\left(G_{h}\right)$ matrices will not change but the adjacent $A\left(G_{h}\right)$, incident $I\left(G_{h}\right)$, volume $V\left(G_{h}\right)$, vertex volume $N_{h}\left(G_{h}\right)$ and edge volume $J\left(G_{h}\right)$, area volume $U_{h}\left(G_{h}\right)$ matrices will change, where $F_{6 m}\left(l_{j h}^{0}\right)=l_{(j-1) h}^{0}$, where $j=0,1,2 \ldots \infty, m=1, \ldots n$.

See.Fig.(7).

such that

$$
\begin{aligned}
& * A_{h}\left(G_{h}\right)=\left[\begin{array}{llll}
0_{012 \ldots \infty h} & 1_{012 \ldots \infty h} & 0_{012 \ldots \infty h} & 0_{012 \ldots \infty h} \\
1_{012 \ldots \infty h} & 0_{012 \ldots \infty h} & 1_{012 \ldots \infty h} & 1_{012 \ldots \infty h} \\
0_{012 \ldots \infty h} & 1_{012 \ldots \infty h} & 0_{012 \ldots \infty h} & 0_{012 \ldots \infty h} \\
0_{012 \ldots \infty h} & 1_{012 \ldots \infty h} & 0_{012 \ldots \infty h} & 0_{012 \ldots \infty h}
\end{array}\right] \stackrel{F_{61}}{\longrightarrow} \\
& A_{h}\left(F_{61}\left(G_{h}\right)\right)=\left[\begin{array}{llll}
0_{012 \ldots \infty h} & 1_{012 \ldots \infty h} & 0_{012 \ldots \infty h} & 0_{012 \ldots \infty h} \\
1_{012 \ldots \infty h} & 0_{012 \ldots \infty h} & 1_{012 \ldots \infty h} & 1_{012 \ldots \infty h} \\
0_{012 \ldots \infty h} & 1_{012 \ldots \infty h} & 0_{012 \ldots \infty h} & 0_{012 \ldots \infty h} \\
0_{012 \ldots \infty h} & 1_{012 \ldots \infty h} & 0_{012 \ldots \infty h} & 0_{012 \ldots(\infty-1) h}
\end{array}\right] \\
& \underset{\lim F_{6 n}}{\longrightarrow} A_{h}\left(\lim F_{6 n}\left(G_{h}\right)\right)=\left[\begin{array}{llll}
0_{012 \ldots \infty h} & 1_{012 \ldots \infty h} & 0_{012 \ldots \infty h} & 0_{012 \ldots \infty h} \\
1_{012 \ldots \infty h} & 0_{012 \ldots \infty h} & 1_{012 \ldots \infty h} & 1_{012 \ldots \infty h} \\
0_{012 \ldots \infty h} & 1_{012 \ldots \infty h} & 0_{012 \ldots \infty h} & 0_{012 \ldots \infty h} \\
0_{012 \ldots \infty h} & 1_{012 \ldots \infty h} & 0_{012 \ldots \infty h} & 0_{01 h}
\end{array}\right], \\
& * V_{h}\left(G_{h}\right)=\left[0_{012 \ldots \infty h}\right] \stackrel{F_{61}}{\longrightarrow} V_{h}\left(F_{61}\left(G_{h}\right)\right)=\left[0_{012 \ldots(\infty-1) h}\right] \\
& \stackrel{\lim F_{6 n}}{\longrightarrow} V_{h}\left(\lim F_{6 n}\left(G_{h}\right)\right)=\left[0_{01 h}\right], \\
& * I_{h}\left(G_{h}\right)=\left[\begin{array}{ccc}
1_{012 \ldots \infty h} & 0_{012 \ldots \infty h} & 0_{012 \ldots \infty h} \\
1_{012 \ldots \infty h} & 1_{012 \ldots \infty h} & 1_{012 \ldots \infty h} \\
0_{012 \ldots \infty h} & 1_{012 \ldots \infty h} & 0_{012 \ldots \infty h} \\
0_{012 \ldots \infty h} & 0_{012 \ldots \infty h} & 1_{012 \ldots \infty h}
\end{array}\right] \stackrel{F_{61}}{\longrightarrow} \\
& I_{h}\left(F_{61}\left(G_{h}\right)\right)=\left[\begin{array}{ccc}
1_{012 \ldots \infty h} & 0_{012 \ldots \infty h} & 0_{012 \ldots \infty h} \\
1_{012 \ldots \infty h} & 1_{012 \ldots \infty h} & 1_{012 \ldots \infty h} \\
0_{012 \ldots \infty h} & 1_{012 \ldots \infty h} & 0_{012 \ldots \infty h} \\
0_{012 \ldots \infty h} & 0_{012 \ldots \infty h} & 1_{012 \ldots(\infty-1) h}
\end{array}\right]
\end{aligned}
$$




$$
\begin{aligned}
& \stackrel{. \lim F_{6 n}}{\longrightarrow} I_{h}\left(\lim F_{6 n}\left(G_{h}\right)\right)=\left[\begin{array}{ccc}
1_{012 \ldots \infty h} & 0_{012 \ldots \infty h} & 0_{012 \ldots \infty h} \\
1_{012 \ldots \infty h} & 1_{012 \ldots \infty h} & 1_{012 \ldots \infty h} \\
0_{012 \ldots \infty h} & 1_{012 \ldots \infty h} & 0_{012 \ldots \infty h} \\
0_{012 \ldots \infty h} & 0_{012 \ldots \infty h} & 1_{01 h}
\end{array}\right], \\
& * N_{h}\left(G_{h}\right)=\left[\begin{array}{c}
0_{012 \ldots \infty h} \\
0_{012 \ldots \infty h} \\
1_{012 \ldots \infty h} \\
0_{012 \ldots \infty h}
\end{array}\right] \underset{F_{61}}{\longrightarrow} N_{h}\left(F_{61}\left(G_{h}\right)\right)=\left[\begin{array}{c}
0_{012 \ldots \infty h} \\
0_{012 \ldots \infty h} \\
1_{012 \ldots \infty h} \\
0_{012 \ldots \infty h}
\end{array}\right] \\
& \underset{\ldots}{\longrightarrow \lim F_{6 n}} N_{h}\left(\lim F_{6 n}\left(G_{h}\right)\right)=\left[\begin{array}{c}
0_{012 \ldots \infty h} \\
0_{012 \ldots \infty h} \\
1_{012 \ldots \infty h} \\
0_{012 \ldots \infty h}
\end{array}\right] \text {, } \\
& *_{h}\left(G_{h}\right)=\left[\begin{array}{c}
0_{012 \ldots \infty h} \\
1_{012 \ldots \infty h} \\
0_{012 \ldots \infty h}
\end{array}\right] \underset{F_{61}}{\longrightarrow} J_{h}\left(F_{61}\left(G_{h}\right)\right)=\left[\begin{array}{c}
0_{012 \ldots(\infty-1) h} \\
1_{012 \ldots(\infty-1) h} \\
0_{012 \ldots(\infty-1) h}
\end{array}\right] \\
& \underset{. . \lim F_{6 n}}{\longrightarrow} J_{h}\left(\lim F_{6 n}\left(G_{h}\right)\right)=\left[\begin{array}{c}
0_{01 h} \\
1_{01 h} \\
0_{01 h}
\end{array}\right], \\
& * U_{h}\left(G_{h}\right)=\left[0_{012 \ldots \infty h} \underset{F_{61}}{\longrightarrow} U_{h}\left(F_{61}\left(G_{h}\right)\right)=\left[0_{012 \ldots(\infty-1) h}\right]\right. \\
& \stackrel{. . \lim F_{6 n}}{\longrightarrow} U_{h}\left(\lim F_{6 n}\left(G_{h}\right)\right)=\left[0_{01 h}\right] .
\end{aligned}
$$

and the 3- chaotic chains are

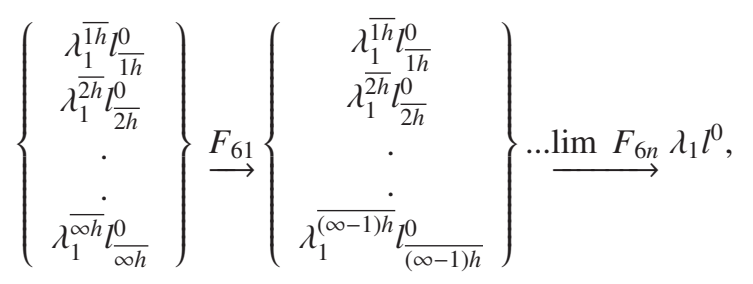

and

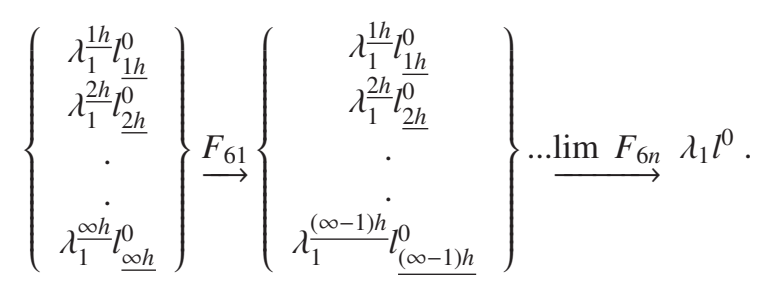

From the above discussion, we will arrive to the following theorems:

\section{Theorem (1):}

The folding of the chaotics into itself which reduce the number of chaotics will induce a folding to the representing matrices and also for the chains.

\section{Proof :}

The prove comes directly from the above cases.

\section{Theorem (2) :}

The folding of the vertices not necessary induce a folding for edges and volumes.

\section{Proof :}

See Fig.(3).and Fig.(4). 


\section{References}

El-Ghoul M. and Mousa Sh. A. (2006). Some geometric transformations on new trees. Journal of mathematics research, vol. 2, Canada.

El-Ghoul M. and Mousa Sh. A. (under press): Chaotic general tree and it's chains. International journal of pure and applied mathematics, Bulgaria.

El-Ghoul M. and Homoda T. (2006). Folding of chaotic multiple graphs and its fractal. International fuzzy mathematics institute, vol. 14, no. 2, USA.

Giblin P. J. (1977). Graphs surfaces and homology.An introduction to algebraic topology. New fetter lane, London EC4P 4EE.

Massey W. S. (1976). Algebraic topology, an introduction, Harcourt, Brace and world Inc., New York, USA.

White A. T. (1973). Graphs, groups and surfaces. Amesterdam, North-Holland publishing Company.

Wilson R. J., Watkins J. J. (1990). Graphs, an introductory approach, a first course in discrete mathematics, Jon Wiley and Sons, Inc., Canada.

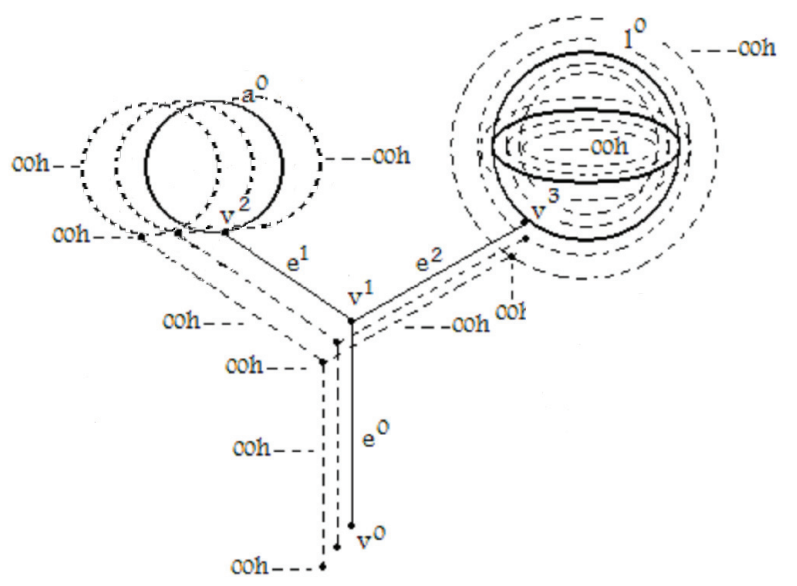

Figure 1.

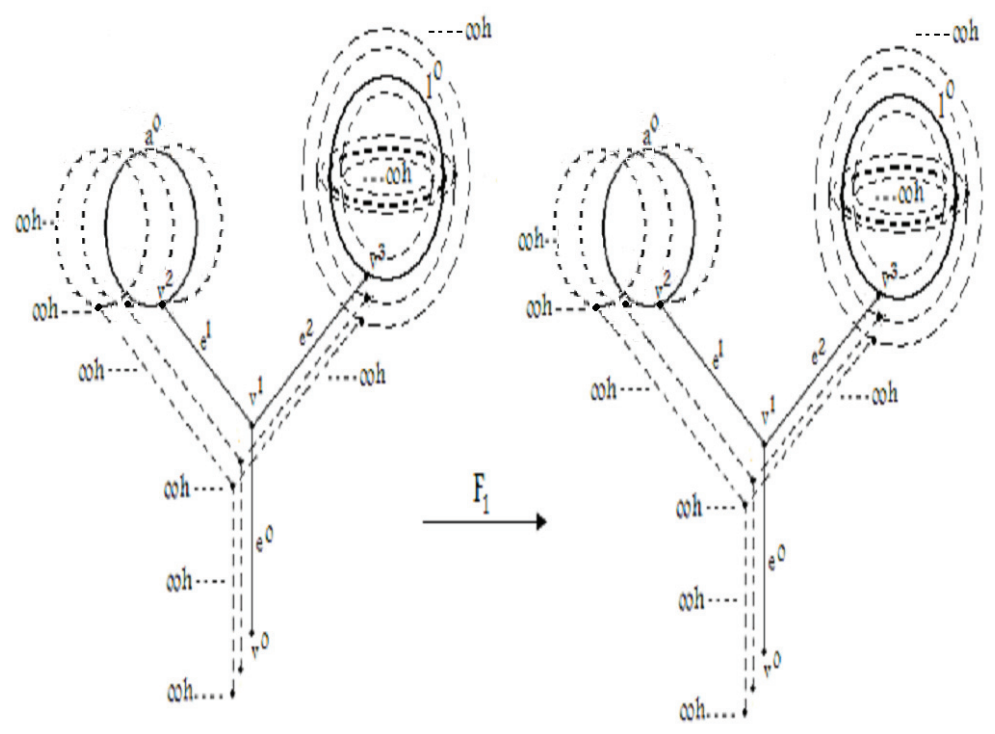

Figure 2. 

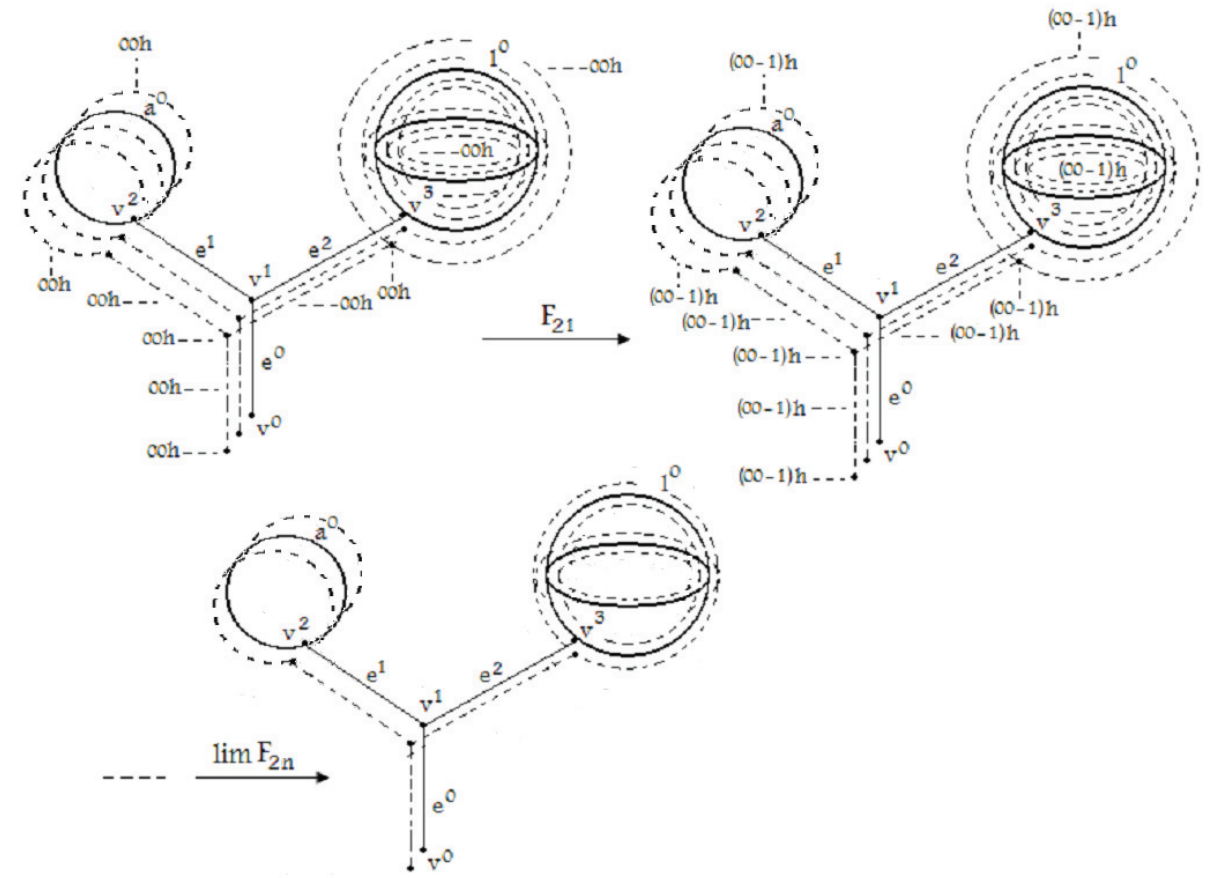

Figure 3.
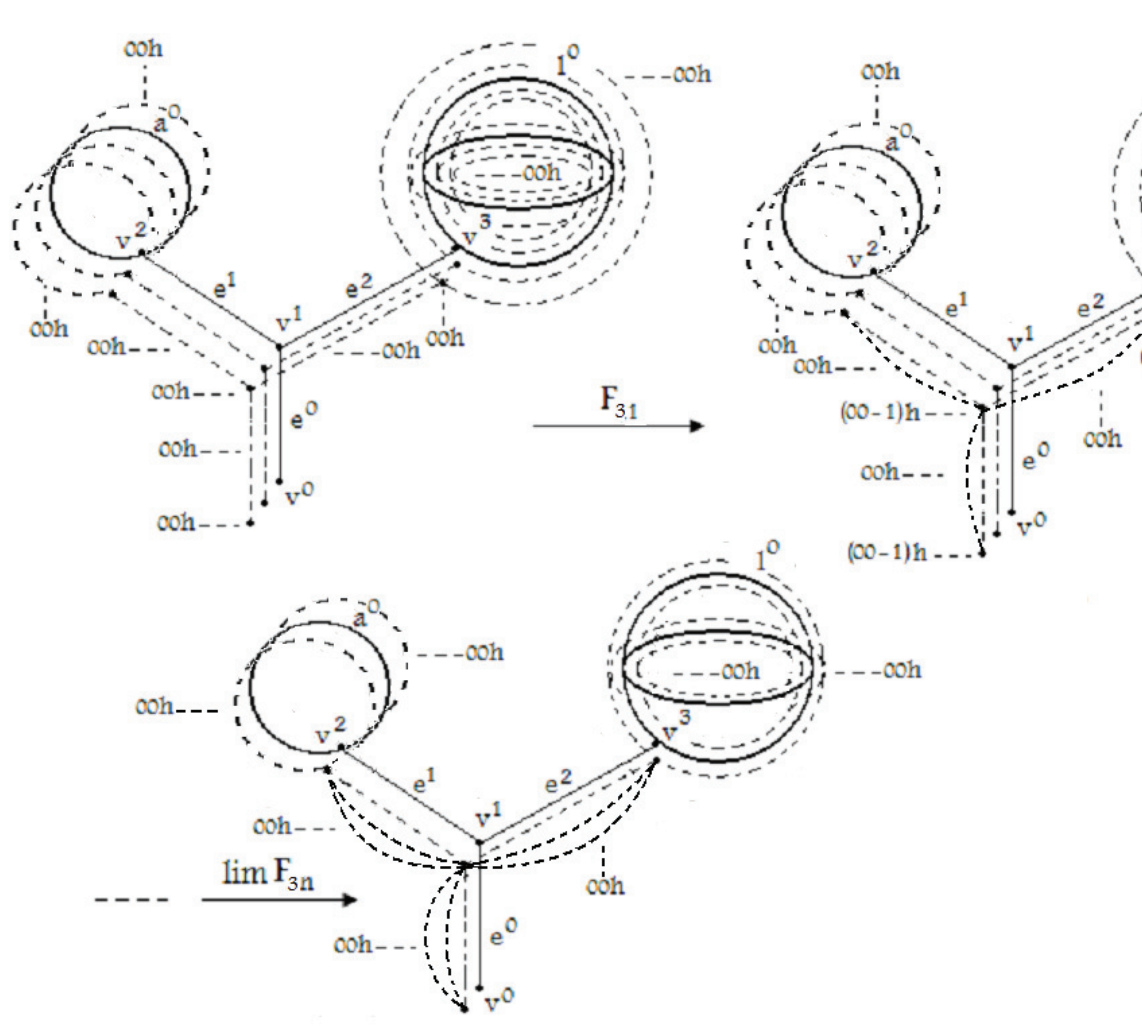

Figure 4. 


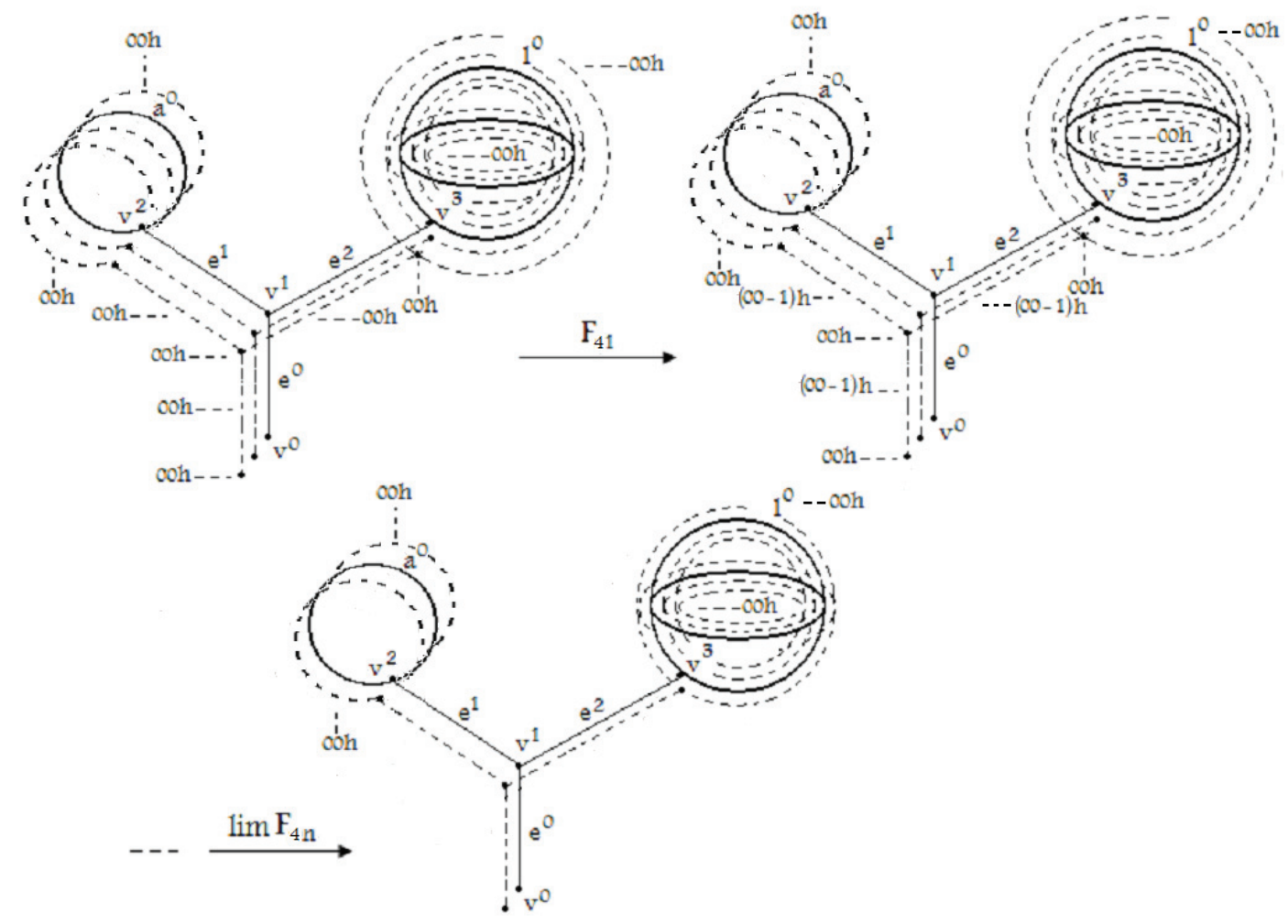

Figure 5.
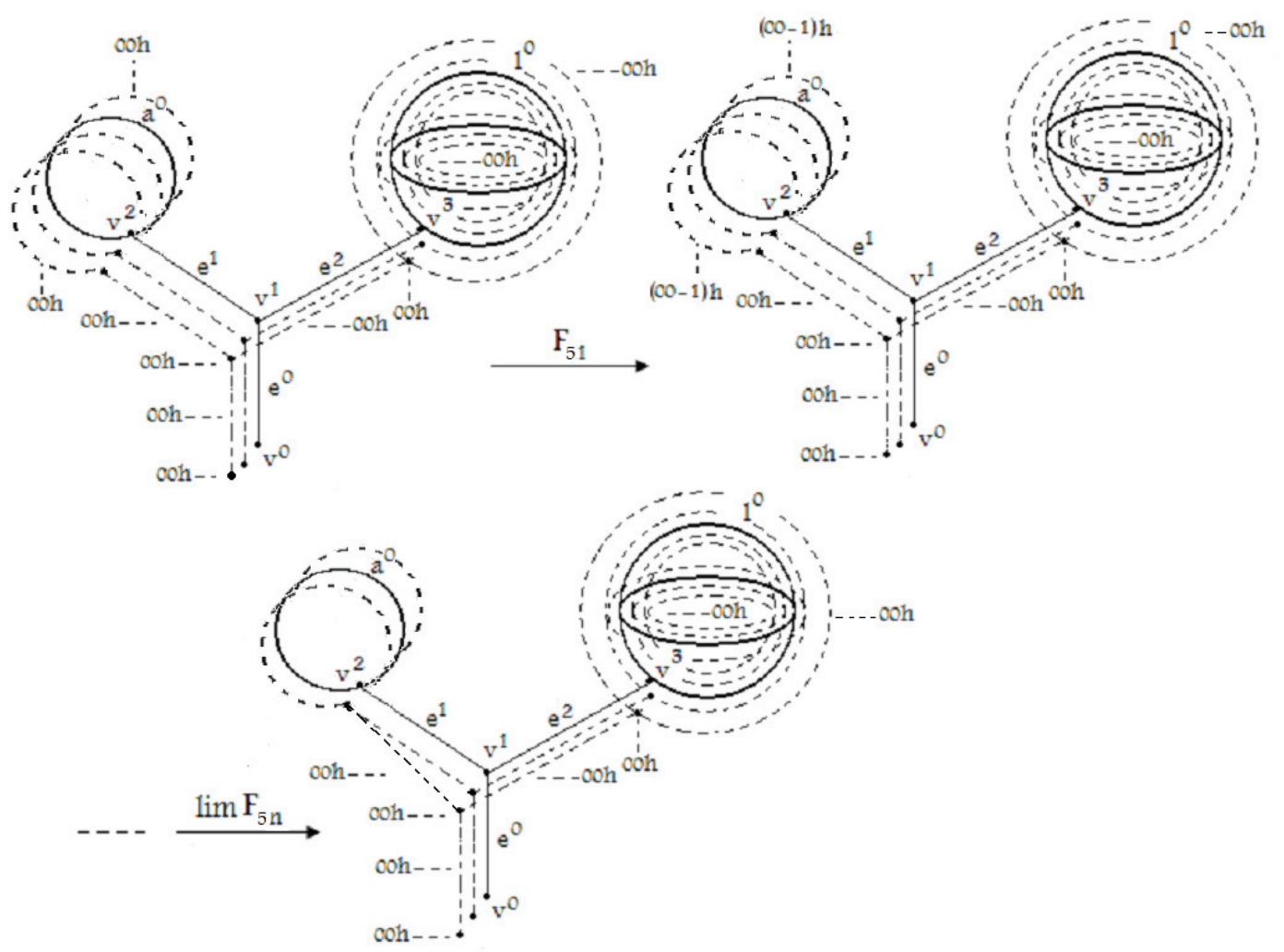

Figure 6. 


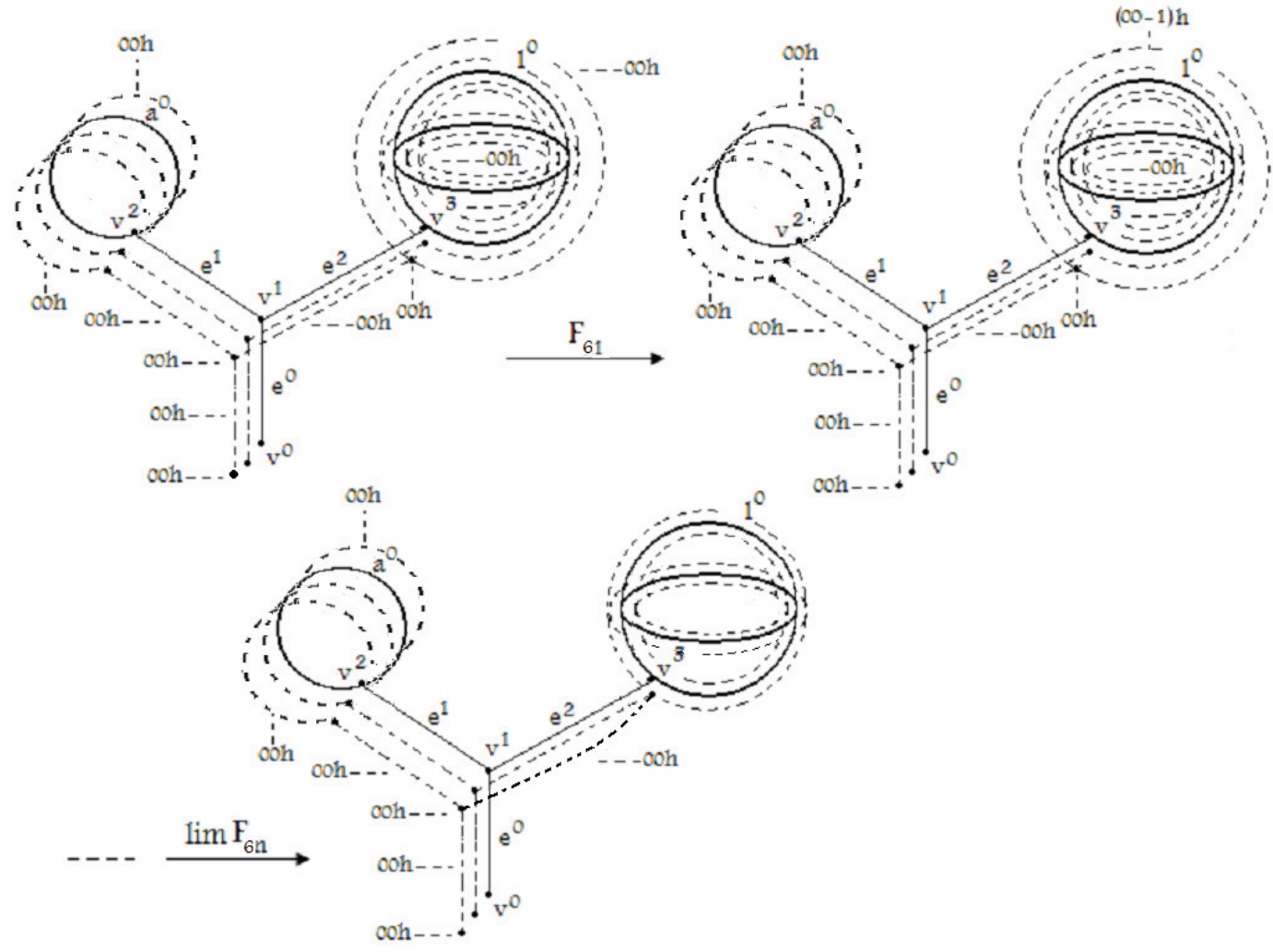

Figure 7. 